\title{
Médiévales
}

Langues, Textes, Histoire

68 | printemps 2015

Langues d'Angleterre

\section{En prévision des vieux jours : les personnes âgées à Montpellier à la fin du Moyen Âge}

Preparing for Old Age: The Elderly in Montpellier at the End of the Middle Ages

Lucie Laumonier

\section{OpenEdition}

Édition électronique

URL : https://journals.openedition.org/medievales/7491

DOI : 10.4000/medievales.7491

ISSN : 1777-5892

Éditeur

Presses universitaires de Vincennes

Édition imprimée

Date de publication : 15 juin 2015

Pagination : 119-146

ISBN : 978-2-84292-430-0

ISSN : 0751-2708

Référence électronique

Lucie Laumonier, «En prévision des vieux jours : les personnes âgées à Montpellier à la fin du Moyen Âge », Médiévales [En ligne], 68 | printemps 2015, mis en ligne le 15 juin 2017, consulté le 22 avril 2022. URL : http://journals.openedition.org/medievales/7491; DOI : https://doi.org/10.4000/medievales. 7491

Tous droits réservés 



\section{Lucie Laumonier}

\section{En prévision des vieux jours : les personnes âgées à Montpellier à la fin du Moyen Âge}

Malgré une présence de plus en plus importante des personnes âgées dans les villes à la fin du Moyen Âge, les études sur la vieillesse en contexte urbain sont peu nombreuses ${ }^{1}$. L'histoire de la vieillesse au Moyen Âge est encore principalement une histoire de ses représentations dans les sources littéraires, médicales ou morales, qui témoignent d'une image plutôt ambivalente de la figure du vieillard ${ }^{2}$. Pourtant, les archives dites «de la pratique» permettent d'éclairer les conditions de vie des personnes âgées dans les villes des $X \mathrm{XV}^{\mathrm{e}}$ et $\mathrm{XV}^{\mathrm{e}}$ siècles, invitant à une réflexion sur la place qu'elles occupent et qui leur est faite ${ }^{3}$. À cet égard, les travaux menés

1. Pour un bilan des différentes études menées en démographie historique, pour la France, l'Italie et l'Angleterre, $c f$. D. Youngs, The Life Cycle in Western Europe, c. 13001500, Manchester, 2006, p. 30-32. L'augmentation de la part de personnes âgées à la fin du Moyen Âge s'explique sans doute par l'accroissement des décès des jeunes en période d'épidémie (ibid., p. 31).

2. Cf. M.-T. Lorcin, «Vieillesse et vieillissement vus par les médecins du Moyen Âge», Bulletin du Centre d'histoire économique et sociale de la région lyonnaise, 4 (1983), p. 5-22; G. Minois, Histoire de la vieillesse en Occident. De l'Antiquité à la Renaissance, Paris, 1987, plusieurs chapitres concernent le Moyen Âge; Vieillesse et vieillissement au Moyen Âge, Senefiance, 19 (1987); M. M. SheEhan éd., Aging and the Aged in Medieval Europe, Toronto, 1990; L. CARruthers et A. PAPAhagi éd., Jeunesse et vieillesse. Images médiévales de l'âge en littérature anglaise, Paris, 2005. Voir aussi les études consacrées aux âges de la vie, telles que H. Dubois et M. ZINk éd., Les Âges de la vie au Moyen Âge, Paris, 1992; ou D. Youngs, The Life Cycle... Plus récemment, sur l'Italie du XV ${ }^{\mathrm{e}}$ au XVII ${ }^{\mathrm{e}}$ siècle, cf. C. Schuster-Cordone, Le Crépuscule du corps. Images de la vieillesse féminine, Fribourg, 2009. On trouvera également quelques éléments sur la vieillesse au Moyen Âge dans des ouvrages généraux comme ceux de V. Gourdon, Histoire des grands-parents, Paris, 2001, ou J. P. Gutton, Naissance du vieillard, Paris, 1988.

3. Voir en particulier la section II de l'ouvrage de M. M. SheEhan éd., Aging and the Aged..., comportant des études très éclairantes de, entre autres, J. RussEl ou D. HerLiHy. Pour la France, $c f$. M.-T. LoRCIN, «Retraite des veuves et filles au couvent. Quelques aspects de la condition féminine à la fin du Moyen Âge», Annales de démographie historique, 1975, 
dans le monde anglophone offrent des pistes à explorer plus en détail à travers une étude monographique, permettant de multiplier les approches documentaires et analytiques ${ }^{4}$. La ville de Montpellier est un espace de choix pour mener une telle recherche, car y sont conservées des sources nombreuses et d'une grande qualité. Ville principale du bas Languedoc, Montpellier se situe dans une région longtemps réputée, selon les historiens du droit, pour son «esprit communautaire», suscitant l'expression vivace des solidarités familiales ${ }^{5}$. En témoigne la fréquence des ménages élargis dans les espaces méridionaux, permettant aux enfants de prendre soin de leurs parents vieillissants. Quand les enfants sont récalcitrants ou font preuve d'une attitude déplaisante à l'égard de leurs parents, la grande liberté testamentaire en vigueur à Montpellier dans la charte de 1204 et l'héritage du droit romain permettent aux personnes âgées d'agiter la menace d'une privation d'héritage, contraignant les plus jeunes à s'occuper d'eux durant leurs vieux jours ${ }^{6}$.

Mais les grandes mortalités de la deuxième moitié du XIV siècle bouleversent un équilibre démographique déjà précaire ${ }^{7}$. À Montpellier, nombreux sont les testateurs sans descendant dont la vieillesse s'annonce difficile en l'absence de bâton sur lequel se reposer. Le cadre citadin est parfois accusé dans l'historiographie d'entraîner un relâchement des solidarités familiales et un isolement toujours plus grand des personnes

p. 187-204, ou F. Autrand, «La force de l'âge: jeunesse et vieillesse au service de l'État en France aux $\mathrm{XIV}^{\mathrm{e}}$ et $\mathrm{XV}^{\mathrm{e}}$ siècles », Comptes rendus des séances de l'Académie des inscriptions et belles-lettres, 129/1 (1985), p. 206-223.

4. Cf. S. Shahar, Growing Old in the Middle Ages. Winter Clothes us in Shadow and Pain, Londres/New-York, 1997; S. ShaHAR, «Old Age in the High and Late Middle Ages », dans P. Johnson et P. Thane éd., Old Age from Antiquity to Post-Modernity, Londres/NewYork, 1998, p. 43-62; P. Thane, Old Age in English History. Past Experiences, Present Issues, Oxford, 2000 ; I. Metzler, A Social History of Disability in the Middle Ages : Cultural Considerations of Physical Impairment, Londres/New York, 2013, chapitre «Ageing », p. 92153.

5. L'expression est de J. Hilaire, Les Régimes des biens entre époux dans la région de Montpellier, du XIII à la fin du XVI siècle. Contribution aux études d'histoire de droit écrit, Thèse de droit, Université de Montpellier, 1956, p. 329. Voir aussi ID., «Vie en commun, famille et esprit communautaire», Nouvelle revue historique de droit français et étranger, 51 (1973), p. 8-52; et R. Aubenas, «La famille dans l'ancienne Provence», Annales d'histoire économique et sociale, 8 (1936), p. 523-540.

6. Plusieurs testaments de Montpellier montrent des exhérédations de descendants, pour des motifs couverts par la Novelle 115 du code Justinien. Si les motifs dépassent le texte de la Novelle, les testateurs s'appuient sur la totale liberté testamentaire montpelliéraine, qui leur permet de laisser leurs biens à qui ils le souhaitent, aussi longtemps que leurs descendants reçoivent la somme de 5 sous, dite «légitime». Cf. L. DE ChARrin, Les Testaments dans la région de Montpellier au Moyen Âge, Ambilly, 1961.

7. Àce sujet, consulter la recherche archéologique, anthropologique et paléopathologique d'É. Crubézy, S. Duchesne et C. Arlaud, La Mort, les morts et la ville : Saint-Côme et SaintDamien, Montpellier, $X^{e}$-XVI siècles, Paris, 2006. 
âgées ${ }^{8}$. La mobilité géographique, les mortalités, l'étroitesse des logements sont autant de limites posées à la prise en charge par la parenté des individus vieillissants. L'analyse des archives de Montpellier montre qu'à la fin du Moyen Âge cette même parenté demeure un recours puissant dans la vieillesse. Lorsqu'il n'y a pas de parents, le milieu social des personnes âgées joue un rôle déterminant dans la manière dont ces dernières pourront organiser leur fin de vie. Aidées par leurs familles et leurs amis, par les possibilités qu'offre le droit privé et par les structures charitables de la ville, les personnes âgées parviennent souvent à contourner les contraintes de la vie urbaine pour s'assurer une vieillesse en compagnie. Toutefois, certaines échappent à ce filet social et se retrouvent pauvres, isolées, particulièrement vulnérables. Ce sont ces stratégies de protection et les moyens mis en œuvre pour pallier la vulnérabilité et la dépendance guettant les personnes âgées qui constituent le cœur de cette recherche, dont l'objectif est d'étudier les différentes expériences de vieillesse en tenant compte du sexe des personnes, de leur situation familiale et de leur degré de fortune, à partir de l'analyse de testaments, d'actes de donation et des registres fiscaux de Montpellier. Avant d'étudier ces mécanismes, une présentation des sources et de la méthode employée permettra de mettre en lumière la manière dont le grand âge est perceptible dans les archives.

\section{La vieillesse dans les archives de Montpellier}

En 1299, au cours d'une enquête réalisée à Montpellier, on auditionne plusieurs témoins de sexe masculin âgés de 70 ans et plus, dont le témoignage est reçu en raison de leur expérience et de leur longue mémoire ${ }^{9}$. L'un d'entre eux, qui a environ 80 ans, est capable de relater des faits remontant à une soixantaine d'années ${ }^{10}$. En 1336, une quinzaine de revendeuses sont auditionnées au cours d'une enquête portant sur l'occupation commerçante

8. Cf. D. Le BlÉvec, La Part du pauvre: l'assistance dans les pays du Bas-Rhône du XII siècle au milieu du XV siècle, Rome, 2000 (CEFR, 265), p. 781 et 783; A.-L. LALlOuetTE, «Les personnes âgées et leurs familles d'après quelques prédicateurs méridionaux des XIII ${ }^{\mathrm{e}}$ et XIV ${ }^{\mathrm{e}}$ siècles », dans Famille et parenté dans la vie religieuse du Midi, Cahiers de Fanjeaux, $\mathrm{n}^{\circ} 43$, Toulouse, 2008, p. 241-261.

9. Cf. A. Germain, Histoire du commerce de Montpellier antérieurement à l'ouverture du Port de Cette, Montpellier, 1861, vol. I, pièce justificative LXIV, p. 326 sq. Par exemple: «Nicolosus de Riverolo, oriundus de Janua, habitator Aquarum Mortuarum, marinarius, etatis, ut dicit, LXX annorum [...]» (p. 350); «Petrus Eguoserii de Mesoa, olim marinarius et piscator, etatis, ut dicit, LXX annorum vel circa [...]» (p. 359). D'autres exemples figurent dans le texte de l'enquête.

10. Le témoin est «Johannes Praderii de Mesoa, homo domini Agatensis episcopi, olim marinarius nunc mercator, etatis ut dicit bene quatuor-viginti annorum [...] dixit se vidisse et audisse, bene sunt sexaginta anni quod [...]. Deinde dixit se vidisse, bene sunt LX anni vel circa, quando [...]» (ibid., p. 355-356). 
de la place de l'Herberie; parmi elles, la plus âgée déclare avoir 70 ans et rapporte des souvenirs remontant soixante années en arrière, du temps où elle était une petite fille ${ }^{11}$. Quelques années auparavant, en 1321, une enquête, diligentée par le Sénéchal de Beaucaire, restitue le témoignage de Johan Bleran, ancien consul de mer $^{12}$. Interrogé au sujet d'événements passés et récents, ses réponses apparaissent vagues, sa mémoire défaillante («de quibus non recordatur, dixit quod non recordatur») ${ }^{13}$. Face à l'accumulation de ces phrases, le notaire s'enquiert alors de son âge: Johan a «80 ans ou environ» et, semble-t-il, ne fait pas un témoin très fiable, sa mémoire commençant à lui échapper ${ }^{14}$. Outre le fait qu'il n'était pas rare d'atteindre les 70 ans à la fin du Moyen Âge ${ }^{15}$, ces témoignages illustrent la division médiévale de la vieillesse en deux phases. La première est une période appelée senectus, au cours de laquelle la personne vieillissante est encore en pleine capacité mais se retire progressivement de la vie publique - et constitue un témoin de première importance ${ }^{16}$. La deuxième phase est nommée senium; elle correspond au moment où la personne âgée perd ses facultés mentales et physiques pour rejoindre, disent les médecins, l'état humoral de l'enfant. Il n'y a pas d'âge auquel s'effectue la transition entre senectus et senium. Certains individus décèdent sans connaître la deuxième phase $^{17}$.

11. Montpellier, AM, Grand chartrier, Louvet $234, f^{\circ} 98 \mathrm{v}-101 \mathrm{v}$, témoignage de Johanna Poitala. Cette enquête est citée et longuement analysée dans K. ReYERSON, "Les réseaux économiques entre femmes à Montpellier (fin XIII ${ }^{\mathrm{e}}-\mathrm{mi}$ XIV ${ }^{\mathrm{e}}$ siècle)», dans L. Galano et L. LAUMONIER éd., Montpellier au Moyen Âge, bilan et approches nouvelles, à paraître en 2015-2016.

12. «Johannes Bleranii de Montepessulano, oriundus et habitator, testis juratis [...] prima dixit quod bene sunt. XL. anni vel. XLV. vel circa quod ipse fuit consul maris [...]» (Montpellier, AM, HH 279, f० 47-48). Enquête menée entre 1321 et 1323 concernant les consuls de mer de Montpellier, qui ont pris les armes et commis diverses exactions à Carnon et autour des étangs de la région. Merci à Lucie Galano, doctorante à l'Université Montpellier 3 , qui a eu la gentillesse de m'indiquer cette référence.

13. Par exemple: «Requisitus de mense, die et de presentibus quando fuit per dictum stagnum et amovit illa que supra dixit, dixit se non recordari ut supra dixit. Requisitus de hora, dixit quod de die» (Montpellier, AM, HH 279, $\mathrm{f}^{\circ} 47 \mathrm{v}^{\circ}$ ).

14. "Requisitus cujus etatis es ispe tempus, dixit quod bene es etatis IIII $^{\mathrm{xx}}$ annorum vel circa» (ibid., $\mathrm{f}^{\circ} 47 \mathrm{v}^{\circ}$ ). Une belle analyse de la manière de dire l'âge dans le cadre de la vieillesse est à trouver dans D. LETT, Un procès de canonisation au Moyen Âge. Essai d'histoire sociale. Nicolas de Tolentino, 1325, Paris, 2008, p. 190 sq.

15. Cf. D. Youngs, The Life Cycle in Western Europe..., section «Age and Life Expectancy», p. 11-39 (p. 26); J. ForGENG, Daily Life in Medieval Europe, Wesport, 1999, p. 30-31. On ne dispose d'aucune donnée à Montpellier sur l'espérance de vie.

16. $C f$. B. RiвÉmont, «Quelques aspects de la relation vieillesse/sagesse au Moyen Âge, l'exemple du Chevalier au barisel», dans Vieillesse et vieillissement..., p. 299-316.

17. $C f$. M.-T. Lorcin, "Gérontologie et gériatrie au Moyen Âge», dans Vieillesse et vieillissement..., p. 199-213 (en particulier p. 205). 
À l'exception des enquêtes et des archives judiciaires, rares à Montpellier, les sources médiévales qui indiquent l'âge des individus sont peu nombreuses. Cette difficulté prévaut dans toutes les études tournées vers une classe d'âge, et ne fait pas exception pour la vieillesse. Il faut alors prendre en considération le fait que la notion d'âge est aussi subjective et culturelle, et qu'elle ne s'appuie pas uniquement sur un chiffre ${ }^{18}$. Une grande variété d'âges est avancée par les rédacteurs de traités et d'encyclopédies médiévales pour donner des bornes à la vieillesse. En fonction des auteurs, elle peut commencer vers 40 ans, 50 ans, 60 ans ou 70 ans: sous la plume du médecin montpelliérain Bernard de Gordon, par exemple, la vieillesse («aetas senectutis ») démarre à partir de $35 \mathrm{ans}^{19}$. Les études sur les représentations de la vieillesse sont stimulantes car elles lui assignent une place spécifique sur l'arbre de parenté. Dans ces représentations, la quintessence de la bonne vieillesse s'incarne dans l'image valorisée du patriarche, de l'aïeul éclairé, qui se retire à la fin de sa vie dans les ordres ${ }^{20}$. La mauvaise vieillesse est portée principalement par l'image d'une femme souvent seule, rusée et manipulatrice, désormais infertile ${ }^{21}$. Malgré la dualité homme/femme, ces deux portraits ont en commun le fait que le patriarche, comme la vieille femme, appartiennent à une génération pouvant avoir plusieurs niveaux de descendants, un moyen d'identifier les personnes âgées dans les archives de la pratique.

\section{Les contribuables et testateurs âgés de Montpellier}

Un premier corpus de sources est constitué par les registres fiscaux de la ville, les «compoix». Ils sont conservés pour le Moyen Âge de 1380 à 1480 et totalisent une quarantaine de registres ${ }^{22}$. Ils servent aux consuls

18. Pour une illustration, $c f$. C. Gauvard, «Les jeunes à la fin du Moyen Âge: une classe d'âge? », dans Les Entrées dans la vie. Initiations et apprentissages, $12^{\circ}$ congrès de la SHMESP, Nancy, 1982, p. 225-244.

19. Les médecins s'accordent sur le fait que, physiologiquement, elle se caractérise par une faiblesse physique croissante, parfois aussi mentale, attribuable au refroidissement et à l'assèchement des humeurs, en particulier l'humide radical: $c f$. M. McVAUGH, «The Humidum Radicale in Thirteenth Century Medicine», Traditio, 30 (1974), p. 259-283. Sur la perception médicale de la vieillesse, voir aussi L. Demartre, «The Care and Extension of Old Age in Medieval Medicine», dans M. M. SheEhan éd., Aging and the Aged..., p. 3-22.

20. $C f$. A.-L. Lallouette, «Les personnes âgées et leurs familles...».

21. Ce portrait est contrebalancé par des représentations plus positives: voir en particulier J. Agrimi et C. CRisciani, «Savoir médical et anthropologie religieuse. Les représentations et les fonctions de la vetula (XIII ${ }^{\mathrm{e}} \mathrm{XV} \mathrm{v}^{\mathrm{e}}$ siècle)», Annales E.S.C., 48/5 (1993), p. 1281-1308; E. JuÀrez-Almendros, «Aging Women and Disability in Early Modern Spanish Literature», dans J. Eyler éd., Disability in the Middle Ages. Reconsiderations and Reverberations, Farnham, 2010, p. 197-208.

22. Ils sont conservés aux archives municipales de Montpellier à partir de la cote Joffre 239. Pour une présentation détaillée de cette source, $c f$. L. LAUMONIER, «Les compoix 
de Montpellier pour la levée d'un impôt proportionnel à la fortune des ménages et indiquent la composition de la fortune des feux imposables de la ville, exprimée en livres fiscales, impôt auquel s'ajoute une deuxième taxe, personnelle celle-là. La déclaration d'un ménage, appelée "manifeste», est portée par un chef de feu, de préférence un homme. Si d'autres membres du ménage y possèdent des biens, leurs noms sont aussi indiqués avec, souvent, la mention de la relation qui les lie au chef de feu. L'évocation de ce lien permet d'estimer la classe d'âge des individus, montrant par exemple un couple marié bien installé dans la vie, cohabitant avec la mère veuve du mari ou de l'épouse. La mère est alors considérée comme une personne âgée ou vieillissante, puisque, en l'absence d'indication d'âge, c'est la situation d'un individu au sein d'un arbre de parenté qui nous permet de supposer sa vieillesse.

La déclinaison de l'identité des chefs de feu invite à une démarche similaire. Les femmes en particulier sont identifiées par les autorités consulaires grâce à des liens de parenté ${ }^{23}$. Épouses, veuves, filles, mères, voire belles-mères, l'évocation de ces relations permet parfois de les inclure dans une catégorie d'âge spécifique. «Fille de» les rapproche de la jeunesse, tandis que «mère de» nous paraît être un indicateur d'un âge vieillissant ${ }^{24}$. Si le rédacteur du registre inscrit «mère», c'est que la femme est veuve, mais aussi que l'enfant évoqué (le plus souvent un fils) est désormais assez âgé pour avoir valeur de référent. La contribuable est mère d'un homme adulte, elle appartient désormais à une génération ascendante. Plus rarement, un contribuable est qualifié de «vieux» ou d' "impotent» par le notaire. En 1417, Remon Belluoc, laboureur, jure qu'il ne possède rien, «quar es mout viel e paubre home ${ }^{25}$ ». Ce type d'évocation est très sporadique: une quinzaine pour environ 9000 déclarations fiscales.

En dépit de leurs nombreux intérêts, les compoix de Montpellier ne permettent pas d'évaluer de manière satisfaisante la part de contribuables âgés: sur 9347 déclarations fiscales datées de 1380 à 1480 , seulement $1,6 \%$ incluent une personne probablement âgée ou vieillissante (155 cas). Cette proportion très basse s'explique par le fait que seuls les noms

montpelliérains: approche qualitative des archives fiscales médiévales», Memini. Travaux et documents, 14 (2010), p. 97-122.

23. Pour un détail, registre par registre, $c f$. L. LAumonier, Vivre seul à Montpellier à la fin du Moyen Âge, thèse de doctorat, Université de Sherbrooke et Université Montpellier 3, 2013, annexe II.

24. Il convient de souligner que «fille de» est aussi employé pour les femmes adultes et célibataires. Le célibat est cependant très rare, l'expression renvoie surtout à des jeunes filles et jeunes femmes pas encore mariées. Cette méthode est employée avec succès par A. Musin et É. Mertens de Wilmars dans leur étude sur la jeunesse : «'Consideré son joesne eaige...'. Jeunesse, violence et précarité sociale dans les Pays-Bas bourguignons et habsbourgeois ( $\mathrm{XIV}^{\mathrm{e}}-\mathrm{XVI}^{\mathrm{e}}$ siècles)», Revue d'histoire de l'enfance «irrégulière», 9 (2007), p. 25-46.

25. Montpellier, AM, Joffre 245, compoix des Faubourgs, 1417, $\mathrm{f}^{\circ} 27 \mathrm{v}^{\circ}$. 
des propriétaires des biens d'un ménage sont indiqués et que peu de renseignements permettent de les identifier. On ne connaît donc qu'une infime proportion des contribuables âgés de la ville.

La deuxième partie du corpus de sources est composée d'actes notariés. Montpellier est une ville à l'activité notariale intense: les fonds départementaux conservent plusieurs centaines de registres allant des années 1330 au tournant des $\mathrm{XV}^{\mathrm{e}}$ et $\mathrm{XVI}^{\mathrm{e}}$ siècles $^{26}$. De nombreux actes antérieurs concernant les habitants de la ville sont à rechercher dans le fonds municipal des ouvriers de la Commune Clôture, chargés dès le début du XIII ${ }^{\mathrm{e}}$ siècle de l'entretien des murailles de Montpellier et investis par certains habitants du rôle d'exécuteurs testamentaires ou de patrons de chapellenies ${ }^{27}$. Dans ces fonds notariés, pour une période allant de 1250 à la fin du $\mathrm{Xv}^{\mathrm{e}}$ siècle, 564 testaments ont été dépouillés. Dans ces actes, c'est la mention d'une parenté descendante de deuxième génération qui suggère l'appartenance d'un testateur à la catégorie «personne âgée». Un legs aux petits-enfants, aux petits-neveux et aux petites-nièces indique que le testateur se situe en position d'aïeul dans sa parenté. Cette méthode induit que les personnes âgées identifiées ont nécessairement des parents, avec lesquels ils résident le plus souvent. Parmi les 564 testaments, quarante-quatre ont été dictés par des Montpelliérains vieillissants ou âgés, ce qui représente 7,8\% du total ${ }^{28}$.

Dans les testaments du Gévaudan du Xve siècle, Philippe Maurice a observé une part de personnes âgées s'élevant à $14,3 \%{ }^{29}$. La méthode employée par le chercheur a inspiré la nôtre: il calcule le nombre de générations séparant le testateur de sa lignée descendante. À Florence et dans son contado en 1427, plus de $20 \%$ des habitants sont âgés de 60 ans et plus $^{30}$. En Angleterre aux XIV et XV ${ }^{\mathrm{e}}$ siècles, entre 10 et $12 \%$ de la population

26. Montpellier, Archives départementales de l'Hérault (désormais ADH), série 2 E 95. Des actes antérieurs sont conservés dans la série G. Environ 7,5\% du fonds a été dépouillé, soit 35 registres. D'autres actes proviennent des fonds municipaux (série BB, série Louvet).

27. Montpellier, AM, série EE. Cf. A. Montel, «Le catalogue des Chapellenies », Revue des langues romanes, 3 (1872), p. 292-294; ID., «Catalogue des Chapellenies, suite et fin», Revue des langues romanes, 4 (1873), p. 5-43. Le fonds a été intégralement inventorié, permettant un accès plus aisé aux documents: $c f$. M. Oudot DE DAINVILle et M. Gouron, Inventaire des archives de la ville de Montpellier, t. XII, Sous-série EE, archives de la commune clôture et des affaires militaires, Montpellier, 1974.

28. Dans sa thèse, Cécile Béghin-Le Gourriérec propose une estimation de 5,5\% de grands-parents parmi 403 testaments montpelliérains datés des XIV et $\mathrm{XV}^{\mathrm{e}}$ siècles: cf. C. BéGHIN-Le Gourriérec, Le Rôle économique des femmes dans la sénéchaussée de Beaucaire à la fin du Moyen Âge (XIV -XV siècles), thèse de doctorat, EHESS, 2000, annexe 3.4, tableau 7.

29. P. Maurice, La Famille en Gévaudan au Xve siècle (1380-1483), Paris, 1998, p. 78.

30. D. Herlihy et C. Klapisch-Zuber, Les Toscans et leurs familles: une étude du « catasto » florentin de 1427, Paris, 1978, p. 656-663. 
seraient des personnes âgées ${ }^{31}$. Les estimations pour Montpellier paraissent alors particulièrement basses ${ }^{32}$. Le taux de 7,8\% dans les testaments doit sans doute être considéré comme un minimum car il n'inclut, on le rappelle, que les testateurs ayant mentionné des petits-enfants, des petits-neveux ou des petites-nièces.

\section{Des femmes âgées en famille : le miroir déformant des sources}

Les personnes âgées et vieillissantes des archives de Montpellier, qu'elles soient des contribuables ou des testateurs, sont plutôt des femmes. Dans les registres fiscaux, ces dernières représentent $55 \%$ des contribuables âgés, alors qu'elles ne constituent en moyenne que $14 \%$ des contribuables ${ }^{33}$. Les testateurs de Montpellier se partagent entre $60 \%$ d'hommes et $40 \%$ de femmes, mais dans le cas des personnes âgées, les testatrices constituent $75 \%$ de l'effectif ${ }^{34}$. Les femmes sont donc surreprésentées dans les rangs des personnes âgées. Ce particularisme sexué de la vieillesse est-il attribuable aux archives elles-mêmes ou témoigne-t-il d'une réalité démographique de la vieillesse à Montpellier? Car, en Gévaudan, la proportion de testatrices âgées demeure stable vis-à-vis de la proportion de testatrices ${ }^{35}$. Dans les registres fiscaux, la différence de distribution entre contribuables femmes et contribuables femmes âgées s'explique par le système d'identification sexué employé par le notariat consulaire de Montpellier, système qui ne lui est pas exclusif ${ }^{36}$. Si plus de $70 \%$ des femmes sont identifiées en fonction d'un lien de parenté, ce n'est le cas que d'environ $5 \%$ des hommes, préférablement désignés par leur titre professionnel. Comme c'est par la position occupée au sein du foyer que l'on peut identifier des personnes âgées, les femmes, qualifiées plus souvent que les hommes par ces liens, sont avantagées par la méthode.

Quant aux testaments, au lieu de suggérer que les femmes sont plus nombreuses à être âgées que les hommes, ils indiquent que les femmes

31. C'est-à-dire âgées de 60 ans et plus. $C f$. J. Russel «How Many of the Population were Aged?», dans M. M. SHEeHan (éd.), Aging and the Aged..., p. 119-128 (p. 123-124).

32. Pour autant, elles sont supérieures à celles de Reims en 1422 (entre 5,2 \% et 6,3\% de personnes âgées). Ibid., p. 125.

33. 85 femmes, 69 hommes, un couple.

34. 33 femmes et 11 hommes.

35. P. Maurice, La Famille en Gévaudan..., p. 78.

36. Le cas parisien: $c f$. C. Bourlet, «L'anthroponymie à Paris à la fin du XIII ${ }^{\mathrm{e}}$ siècle d'après les rôles de la taille du règne de Philippe Le Bel », dans M. Bourin et P. ChareiLle éd., Genèse médiévale de l'anthroponymie moderne, Tours, t. II-2, 1992, p. 9-44; des cas anglais : $c f$. C. Beattie, «The Problem of Woman's Work Identities in Post Black Death England», dans J. Bothwell, J. Godberg et M. Omrod éd., The Problem of Labour in FourteenthCentury England, York, 2000, p. 1-19. 
testent à un âge plus avancé que les hommes ${ }^{37}$. Cela s'explique en grande partie par le fonctionnement du système dotal et par la préférence accordée aux garçons pour hériter des biens. Règne à Montpellier une complète liberté testamentaire: tant que les enfants reçoivent une somme minimale, la «légitime», fixée dans les pratiques à 5 sous, chacun peut instituer qui il souhaite, voire ne pas avoir d'héritier universel. Pourtant, les filles sont minoritairement choisies comme héritières universelles par leurs parents; elles sont le plus souvent dotées et, selon le droit fixé dans la charte de coutumes, exclues de la succession ${ }^{38}$. Bien qu'elles disposent de paraphernaux et reçoivent souvent des dons testamentaires de la part de leurs parents - en plus de leur dot -, leur patrimoine est fréquemment géré par leur mari ${ }^{39}$. À la mort de ce dernier, elles disposent de leurs biens avec une certaine liberté, comme en témoigne le fait que plus de la moitié des testatrices sont des veuves ${ }^{40}$. Souvent gratifiées de legs par leur défunt mari, leur héritière universelle dans $40 \%$ des cas, c'est pendant le veuvage que les femmes apparaissent les plus actives dans les sources ${ }^{41}$.

Les hommes testent donc avant et pendant leur mariage car ils disposent plus rapidement et de davantage de biens que les femmes, tandis que ces dernières testent après leur veuvage, lorsqu'elles sont adultes ou âgées ${ }^{42}$. Ces logiques se répercutent sur le profil démographique des testateurs et sont observables dans d'autres espaces à la même période ${ }^{43}$. Les femmes âgées sont alors plus visibles dans la documentation que les hommes âgés, dont les testaments ont été enregistrés plus tôt au cours de leur vie.

Enfin, parce que la méthode employée repose principalement sur l'énonciation des liens de parenté, les personnes âgées de Montpellier paraissent très entourées, fort peu isolées. Sur les 155 déclarations fiscales incluant une personne âgée, $78 \%$ témoignent de ménages élargis. Dans plus de $90 \%$ des cas, le contribuable âgé vit avec un de ses enfants adultes,

37. Les hommes âgés ne constituent que 3,3\% des testateurs de sexe masculin (11 sur 330), tandis que les femmes âgées représentent $14,1 \%$ des testatrices (33 sur 234).

38. Selon les estimations de C. Béghin-Le Gourriérec, entre les années 1250 et 1400, environ $80 \%$ des testateurs qui ont des enfants et $60 \%$ des testatrices qui sont mères choisissent un garçon comme héritier universel. Ce phénomène est accentué au $\mathrm{Xv}^{\mathrm{e}}$ siècle, où il atteint 90 \% (C. BÉGHIN-Le GouRRIÉREC, «Dot, patrimoine et solidarité à Montpellier dans les derniers siècles du Moyen Âge», Études roussillonnaises, 25 (2013), p. 31-40, ici p. 36).

39. $C f$. M.-C. MarandET, «Le patrimoine des femmes en région toulousaine», Études roussillonnaises, 25 (2013), p. 41-50 (p. 50).

40. Les veuves sont au nombre de 130, pour un total de 234 testatrices.

41. Cf. C. BÉGHIN-Le GourRIÉREC, «Dot, patrimoine et solidarité...», p. 39.

42. Ce fait est exacerbé à Florence où environ $75 \%$ des testatrices sont veuves. Voir l'étude d'I. Снавот, La Dette des familles. Femmes, lignage et patrimoine à Florence aux XIV et $X V^{e}$ siècles, Rome, $2011(C E F R, 445)$.

43. Cf. P. Maurice, La Famille en Gévaudan..., p. 89; J.-P. Barraqué, Saragosse à la fin du Moyen Âge. Une ville sous influence, Paris, 1998, p. 170. 
comme Francesa, veuve de Johan Bort, qui réside avec sa fille Perretta et son gendre Denis Melines, dans le quartier Sainte-Foy en $1470^{44}$. Parmi les 44 testateurs âgés, plus de la moitié réside en compagnie, de préférence, de membres de leur parenté, telle Bérauda, veuve de Paul Privat qui vit avec sa petite-nièce Johannetta et avec une femme veuve, dans une maison des faubourgs de la ville ${ }^{45}$. Malgré la présence écrasante du premier cercle de parenté dans la prise en charge de la vieillesse, d'autres formes de cohabitation montrent que les individus vieillissants font appel à leurs parents éloignés ou à des tiers pour s'occuper d'eux, tandis que certains vivent seuls et affrontent parfois avec difficulté leur fin de vie. Cette déclinaison de situations s'articule sur un axe essentiel, la présence d'une parenté autour de la personne âgée. Néanmoins, de nombreux autres paramètres entrent en compte lorsqu'il s'agit de prévoir ses vieux jours, tels que la santé mentale et physique, les besoins des enfants désormais adultes, ainsi que les ressources matérielles ${ }^{46}$.

\section{Vieillir en famille}

Les sources de Montpellier montrent que la parenté consanguine est investie en premier lieu du rôle de soutien pendant la vieillesse. Il existe cependant de multiples manières de vieillir dans le giron familial. Ces différentes situations, ainsi que les processus menant éventuellement des unes aux autres, se comprennent en fonction du rang occupé dans le ménage par les personnes âgées, de la nature des liens qui les unissent à leurs parents, de l'investissement émotionnel de ces relations et des capacités cognitives et physiques des individus. La question de la place de la personne âgée dans le groupe domestique fait partie des préoccupations des prédicateurs méridionaux, qui valorisent fortement la figure du patriarche, idéal de la «bonne vieillesse» masculine, quand elle est vécue dans le siècle ${ }^{47}$. Dans les archives fiscales de la ville méridionale, ce rôle n'est pris que par un tiers des personnes âgées, les deux autres tiers occupant dans le ménage une place secondaire.

44. Montpellier, AM, Joffre 266, compoix de Sainte-Foy, $1470, \mathrm{f}^{\circ} 92 \mathrm{v}^{\circ}$.

45. Montpellier, ADH, 2 E 95-445, Arnaud Vitalis (1412), 13 mai 1412, f 27.

46. «Admittedly the strategies [that peasants devised to cope with the spectre of insecurity in old age] reflected individual needs and preferences, particularly about how to settle problems that ranged from the onset of infirmity to the coming of age of children wanting land in order to marry, to a man's acute weariness after years of hard labour» (E. CLARK, «The Quest for Security in Medieval England», dans M. M. SheEhan éd., Aging and the Aged..., p. 189-200 [p. 191]).

47. Cf. A.-L. Lallouette, «Les personnes âgées et leurs familles... », p. 244. 


\section{La vieillesse flamboyante: patriarche et matriarche}

La position dominante de pater familias implique l'existence d'une familia sur laquelle exercer la patria potestas et d'un patrimoine à gérer ${ }^{48}$. Afin de conserver ce rôle, l'homme vieillissant doit garder l'emprise sur ses biens, car prendre sa retraite comporte un certain nombre de désavantages, en particulier la perte graduelle d'un statut dominant ${ }^{49}$. Garder le contrôle sur les biens et le pouvoir dans le ménage est une recommandation que l'on trouve par exemple dans l'Ecclésiastique ${ }^{50}$. C'est aux hommes, et nullement aux femmes, que les discours des prédicateurs s'adressent lorsqu'ils incitent les vieillards à garder la haute main sur le ménage ${ }^{51}$. Mais si une part importante des Montpelliérains âgés conservant un ascendant sur le groupe domestique sont des hommes, on compte aussi des femmes parmi ces chefs de feu $^{52}$. Un exemple de l'investissement de ce rôle, par un homme et par une femme, se trouve dans le testament, daté du 2 juin 1478, de Martin Rodier, «honnête homme», cultivateur et habitant de Montpellier ${ }^{53}$.

Au vu de son arbre de parenté, Martin est probablement âgé d'une soixantaine d'années. C'est un homme marié, dont l'épouse Agnès est toujours en vie. Le couple a eu au moins deux enfants, un garçon et une fille, qui se sont mariés à leur tour et ont eu eux aussi des enfants. Le fils et la fille de Martin et d'Agnès sont décédés au moment de la rédaction du testament de leur père mais leurs conjoints sont encore en vie. Martin et sa femme Agnès ont trois petits-enfants: Margarida, fille de leur fille, ainsi que Florensa et Barthélémy, tous deux mineurs, nés du mariage de leur fils. Le testateur donne à Margarida la somme de 20 livres, laisse à Florensa 80 livres pour son mariage et l'entretien jusqu'à ses noces. Agnès, l'épouse de Martin, reçoit l'usufruit des biens et la tâche d'en faire la gestion ${ }^{54}$.

48. $C f$. J. Hilaire, «Patria Potestas et pratique montpelliéraine au Moyen Âge. Symbolisme du droit écrit », Mémoires de la Société pour l'histoire du droit et des institutions des anciens pays bourguignons, comtois et romands, 29/1 (1968), p. 421-436. La notion de «familia» ne réfère pas tant ici à la parenté qu'au ménage, au groupe domestique, dont le chef de feu est à la tête: $c f$. C. CAROzZI, «Familia-domus: étude sémantique et historique», dans Famille et parenté dans la vie religieuse..., p. 15-30.

49. «Retirement had its disadvantages and doubtless entailed diminution of status» (S. SHAHAR, «Old Age in the High...», p. 58).

50. «Filio et mulieri, fratri et amico, non des potestatem super te in vita tua: et non dederis alii possessionem tuam, ne forte pœniteat te, et depreceris pro illis»; « $\mathrm{A}$ ton fils, à ta femme, à ton frère, à ton ami, ne donne pas pouvoir sur toi pendant ta vie. Ne donne pas à un autre tes biens, tu pourrais le regretter et devrais les redemander» (Ecclésiastique, 33: 20).

51. Cf. A.-L. LallouEtTE, «Les personnes âgées et leurs familles... ».

52. C'est aussi le cas en Angleterre dans les familles rurales: $c f$. E. CLARK, «The Quest for Security...», p. 191-193.

53. Montpellier, ADH, 2 E 95-458, Arnaud Vitalis (1418-1481), 2 juin 1478, $\mathrm{f}^{\circ} 25 \mathrm{v}^{\circ}$.

54. «Item fecit, ordinavit, instituit et nominavit dominam senhoressam ponderosam et usufructuariam omnium et quorum cumque bonorum suorum, mobilium et inmobilium, 
Aucune mention de sa dot n'est faite dans l'acte ${ }^{55}$. Barthélémy, l'unique petit-fils, est institué héritier universel par son grand-père ${ }^{56}$. La tutelle de Florensa et Barthélémy, tous deux mineurs, revient à Agnès ${ }^{57}$. Si elle décède et que les enfants sont toujours pupilles, leurs tuteurs seront leur père et leur tante. Martin accorde toute confiance à sa femme, en refusant qu'elle rende des comptes de sa gestion aux cours de Montpellier ${ }^{58}$.

Martin Rodier distribue ses biens et règle sa succession en patriarche. Bien que les petits-enfants aient tous un de leurs deux parents en vie, il revient à Martin et à sa femme, aïeuls de la lignée, de prendre en charge leur avenir, garantissant la circulation des biens et l'éducation des plus jeunes. Selon les compoix, lorsqu'un homme vieillissant ou âgé habite dans un groupe domestique étendu, il détient la première place dans la moitié des cas. Un rôle similaire est endossé par les femmes à la mort de leur époux : Agnès recevra ainsi, au décès de son époux, la responsabilité d'administrer ses biens et de prendre soin de ses petits-enfants mineurs. L'accès des femmes à la tête du foyer est perceptible dans les archives, mais dans une proportion moindre que pour les hommes. Dans les compoix, près de $30 \%$ des femmes âgées vivant dans un ménage élargi y occupent le premier rang, tandis que $70 \%$ d'entre elles ont une place secondaire. Une série d'actes, étendus sur les quarante premières années $\mathrm{du} \mathrm{Xv}^{\mathrm{e}}$ siècle, est particulièrement révélatrice des mécanismes permettant aux femmes vieillissantes de se placer et de se maintenir à la tête de leur parenté, jusqu'à un âge avancé.

Dans les années 1400, Marina dels Fans, riche veuve, réside avec sa fille et son gendre, Arnaud Pelagal, épicier-poivrier, dans le riche quartier Saint-Firmin $^{59}$. Arnaud et sa belle-mère Marina ont mis leurs biens en commun et possèdent «tot ensems»une fortune considérable, estimée

presencium et futurorum et illorum rectorem et administratorem videlicet honestam mulierem Agnetem, uxorem suam» (ibid., $\left.\mathrm{f}^{\circ} 26 \mathrm{v}^{\circ}\right)$.

55. Agnès avait peut-être renoncé aux protections que le droit lui accorde concernant sa dot, celle-ci a pu être aliénée: $c f$. C. BÉGHIN-LE GourRIÉREC, «Dot, patrimoine et solidarité... », p. 38.

56. Sur les règles de succession, voir supra et L. DE ChARrin, Les Testaments dans la région de Montpellier...

57. «Tutricem vero curatorem et gubernatorem predictorum liberorum dicti condam Stephano Roderii videlicet Barthelemis et Florencie, nepotis et neptis suorum pupillorum, fecit et nominavit videlicet predictam Agnetem uxorem suam» (Montpellier, ADH, 2 E 95458, Arnaud Vitalis (1418-1481), 2 juin 1478, acte $\mathrm{f}^{\circ} 25 \mathrm{v}^{\circ}$, ici f ${ }^{\circ} 27$ ).

58. «Et noluit ymo [immo] expresse prohibuit et deffendit quod dicta Agneti faciat aliquod inventarium de predictis bonis sue hereditatis nec ad hoc compelli per aliquam curiam... » (ibid.). La cour du bayle est en charge des dossiers de tutelle, tandis que la cour spirituelle de Saint-Firmin, épiscopale, s'occupe des legs pieux et des litiges successoraux de la juridiction de Montpelliéret: $c f$. L. DE Charrin, Les Testaments dans la région de Montpellier..., section II «Contrôle judiciaire », p. 207, et section III «Matières faisant objet d'un contrôle», p. 213.

59. Montpellier, AM, Joffre 239, compoix de Saint-Firmin, 1404, fo 13. 
à 2352 livres fiscales ${ }^{60}$. Le défunt mari de Marina pratiquait lui aussi l'épicerie, une activité très lucrative dans la ville marchande ${ }^{61}$. Pendant trente ans, la veuve et son gendre disparaissent des sources. Un document postérieur indique qu'au cours de cette période le couple d'Arnaud et de la fille de Marina a donné naissance à trois enfants, deux filles d'abord, puis un fils, prénommé Johan ${ }^{62}$. En juillet 1434, Marina dels Fans réapparaît dans les archives, agissant au titre de tutrice de Johan Pelagal son petit-fils, fils et héritier de feu Arnaud Pelagal ${ }^{63}$. Deux ans plus tard, elle est estimée à des fins d'impôts, en son nom et en celui des héritiers d'Arnaud, pour 2412 livres fiscales ${ }^{64}$. Cette somme inclut plusieurs maisons, dont une où elle vit avec «los heretiés de senhor Arnaut Pelagual ${ }^{65}$. Le testament de la veuve d'Arnaud, daté de 1439 , est le dernier concernant cette famille ${ }^{66}$. La veuve mentionne ses deux filles mariées, dont une avec un poivrier, et nomme Johan, son fils toujours mineur, héritier universel. Les exécuteurs testamentaires sont un de ses gendres et sa mère, Marina dels Fans. L'acte est passé «in domo habitationis dictorum Marine et Johannis Pelagalli ${ }^{67} »$.

Le décès d'Arnaud, qui a laissé derrière lui sa femme, ses filles et son fils encore mineur, n'a que peu bouleversé l'ordre domestique. Marina, désormais grand-mère et tutrice de son petit-fils, demeure à la tête du foyer, mais elle occupe dorénavant cette place seule. Dès le mariage de sa fille, et parce qu'elle était déjà veuve à cette époque, elle a partagé avec son gendre les responsabilités patrimoniales et économiques du ménage. En 1439, elle a certainement dépassé les 60 ans. Aïeule et femme d'expérience, elle agit d'une main ferme et assurée. C'est ainsi que les Montpelliéraines accèdent à la direction des biens et de la maisonnée à la faveur du veuvage, en remplaçant

60. L'en-tête de la déclaration indique qu'il s'agit du «manifeste» de «Dona Marina, molher que fonc de senhor Guilhem dels Fancs, espiciare» et de «senhor Arnaut Pelagal, especiayre, a rendut son manifest am sa suegra, dona Marina, tot ensems ». La veuve est peutêtre héritière universelle de son époux et a doté sa fille de ces biens, en retenant l'usufruit et l'administration.

61. Sur ce sujet, $c f$. J. Combes et A.-E. Sayous, «Les commerçants et les capitalistes de Montpellier aux XIII et XIV siècles», Revue historique, 188-189 (1940), p. 341-377; K. ReYERSOn, The Art of the Deal: Intermediaries of Trade in Medieval Montpellier, Leyde, 2002.

62. La composition de la famille d'Arnaud est connue par le testament de sa femme, daté de 1439 (Montpellier, ADH, 2 E 95-551, Jean Valocière l'Aîné (1439), 5 avril 1439, fo 128).

63. Elle loue ses droits sur les étangs de Pérols (Montpellier, ADH, 2 E 95-538, Giraud Girard (1434-1436), 21 juillet 1434, f० 79).

64. Montpellier, AM, Joffre 249, compoix de Saint-Firmin, 1435, $\mathrm{f}^{\circ} 13$.

65. La même année, Marina, toujours en tant que tutrice de Johan, procède à la mise en location de plusieurs biens sur lequel elle possède des intérêts: Montpellier, ADH, 2 E 95-538, Giraud Girard (1434-1436), 24 novembre 1436, f ${ }^{\circ}$ 117; ibid., 28 novembre 1436, $\mathrm{f}^{\circ} 118 \mathrm{v}^{\circ}$; ibid., 20 mars 1436 (a.s.), f $\mathrm{f}^{\circ} 159 \mathrm{v}^{\circ}$.

66. Montpellier, ADH, 2 E 95-551, Jean Valocière l'Aîné (1439), 5 avril 1439, fo 128. L'acte est détrempé, une partie des feuillets est illisible.

67. Ibid., fo $129 v^{\circ}$. 
leur époux comme chef de feu ${ }^{68}$. Mais, au moment du mariage de leurs enfants, elles doivent négocier leur position dans le ménage. Nul doute que la fortune de Marina, en partie héritée de son mari, l'a favorablement aidée dans cette démarche. Le rôle de ces hommes et de ces femmes vieillissants est socialement avantageux, puisque ceux-ci conservent, en entrant dans la vieillesse, la patria et la matria potestas. Loin de les diminuer, la senectus conforte leur position dominante au sein de leur ménage et de leur parenté. Entourés des leurs, ils pourront se retirer de la première place quand la fatigue, la vieillesse et le désir des enfants d'accéder enfin à la direction du foyer les inviteront à passer la main, à prendre leur retraite.

\section{La retraite en famille: occuper une deuxième place}

Environ les deux tiers des personnes âgées qui vivent en compagnie dans les registres fiscaux occupent au sein du groupe domestique une place secondaire ${ }^{69}$. Il s'agit donc de la majorité des ménages montpelliérains comptant une personne âgée ${ }^{70}$. Cette place a une teinte féminine, puisqu'elle concerne $70 \%$ des femmes âgées, mais la moitié des hommes âgés habitant dans un ménage élargi. Le nom de ces individus n'apparaît dans l'entête de l'estimation qu'après celui du chef de feu; ils possèdent des biens appartenant au groupe domestique, mais ne sont pas les représentants de leur ménage. Toujours impliqués, au moins par leur patrimoine, ils se sont retirés de la direction des affaires et de la maisonnée. Des actes notariés qui concernent Peyre Perrucet, tisserand de toiles, montrent comment peut se dérouler ce processus. Le tisserand procède en 1452 à la mise en ordre de ses affaires, avant de s'en retirer. Par le biais d'une donation entre vifs, il laisse tous ses biens à son fils Guilhem, lui aussi tisserand, à condition que ce dernier l'entretienne sur ses biens, dans sa maison ${ }^{71}$. La cohabitation ne se limite pas aux deux hommes et à leurs épouses: deux autres tisserands

68. Cf. C. Béghin-Le Gourriérec, «La tentation du veuvage. Patrimoine, gestion et travail des veuves dans les villes du Bas-Languedoc aux XIve et Xve siècles», dans La Famille, les femmes et le quotidien (XIVe-XVIII $e^{e}$ siècle). Textes offerts à Christiane Klapisch-Zuber, Paris, 2006, p. 163-180. Sur les veuves dans le milieu des affaires, $c f$. K. ReYerson, «La participation des femmes de l'élite marchande à l'économie: trois exemples montpelliérains de la première moitié du XIV ${ }^{\mathrm{e}}$ siècle», Études roussillonnaises, 25 (2013), p. 129-135.

69. Cela concerne $64 \%$ des personnes âgées vivant en compagnie.

70. Sur les soins des parents vieillissants en Angleterre dans les milieux ruraux, $c f$. E. CLARK, «The Quest for Security... », p. 193 sq.; sur l'évolution de la part de personnes âgées «pensionnaires» de leurs enfants vis-à-vis de la proportion de personnes âgées en situation de chef de feu, $c f$. table 1, p. 194.

71. Montpellier, ADH, 2 E-95-549, Antoine Malarippe (1452-1453), 11 octobre 1452, $f^{\circ}$ 69: «Item [...] quod vos dictus Guilhemus Perruceti filius meus debeatis et teneamini ad et per totum tempus vite mee michi sano et egro providere in cibo, potu, vestitu, calciatu et aliis alimentis michi sano et egro, necessariis, bene et suficienter, juxta facultatem bonorum meorum atque vestrorum, et decentiam persone mee. » L'indication de co-résidence est 
vivent dans la maison, dont un qui s'affrère alors avec Guilhem. Dans cet acte, Guilhem rappelle l'entretien qu'il doit à son père et qui concerne désormais le nouveau venu, Félizot de Goys ${ }^{72}$.

En abandonnant la direction des biens et du ménage et en demandant à son fils de prendre soin de lui, Peyre manifeste sa volonté de se retirer de la vie active, en communauté, et, on l'imagine aisément, en gardant un œil sur l'entreprise familiale. Dans de nombreuses situations, les cohabitations intergénérationnelles ne sont pas formalisées par un acte notarié et échappent à la vigilance de l'historien ${ }^{73}$. Jean Hilaire a montré que l'on peut en trouver la trace dans les contrats de mariage ${ }^{74}$ : c'était sans doute le cas d'Arnaud Pelagal et de sa belle-mère Marina dels Fans. Les testaments sont aussi l'espace notarial par excellence où se prévoient les vieux jours et où se constituent parfois des communautés de vie, permettant la retraite d'une personne vieillissante. Toutefois, les donations effectuées dans les testaments ne prennent effet qu'à la mort du testateur: il s'agit de déclarations d'intention et non, comme dans le cas de donations entre vifs, de dispositions prises immédiatement ou entérinant un état de fait préexistant.

Dans son testament daté de 1420, Jacme Morit donne à Jacmeta, sa femme, ses effets personnels, vêtements, bijoux et chapelets, et lui octroie son entretien complet dans la maison de ses héritiers (leurs enfants), aussi longtemps qu'elle reste veuve et vit dans la maison familiale ${ }^{75}$. Ce type de dispositions, assurant au conjoint survivant une «retraite», est pris en très grande majorité par des hommes, pour leur épouse. Parmi les testatrices mariées, quel que soit leur âge, $3 \%$ seulement laissent l'usufruit de leurs biens à leur mari, contre un quart des hommes mariés qui l'attribuent à leur épouse $^{76}$. Là encore la vieillesse féminine apparaît avec plus de netteté que

donnée à la fin de l'acte: «Actum in domo habitationis dictorum patris et filii necnon et Pauleti de Aulays et Felizoti de Goys, etiam textorum telarum Montispessulani» ( $\left.\mathrm{f}^{\circ} 70\right)$.

72. Ibid., 11 octobre 1452, f 70 : «Item plus fuit et est de pacto quod quamdiu dictus Petrus Perruceti, pater mei dicti Guilhemi, vitam ducet in humanis, quod ego et vos dabimus et mistrabimus [sic] atque promidebimus sibi <in dictam domo [sic]>, cibo, potu, vestitu et calciatu et aliis alimentis sibi sano et egro necessariis bene et sufficienter juxta facultatem bonorum meorum atque suorum et decentiam persone sue, necnon solvemus et distribuemus de bonis suis illos viginti mutones auri seu eorem valorem quos ipse retinuit de eisdem bonis suis in donatione quam ipse de eisdem bonis suis michi fecit $[\ldots] »\left(f^{\circ} 71\right)$.

73. C'est «a widespread, unrecorded, customary practice» (P. Thane, Old Age in English History..., p. 80).

74. J. HiLAire, Les Régimes des biens entre époux...

75. Montpellier, ADH, 2 E 95-414, Jean Solages, 28 août 1420, fo 96v, ici fo 97 : «Item volo et ordino quod Jacoba uxor mea habeat victum et vestitum ac calciatum et omna alimenta sua necessaria quandum staberit in viduitate in domo habitationis heredum meorum predictorum et non alibi. »

76. Il est à noter que plus de $60 \%$ des femmes mariées instituent héritier universel leur époux, tandis que $35 \%$ des testateurs mariés instituent leur épouse. Ces derniers préfèrent 
celle des hommes, car les femmes disposent moins de biens immeubles et ont un accès plus réduit au numéraire que leur mari ${ }^{77}$. Pour cette raison, ce sont eux qui veillent à préparer la fin de vie de leur conjointe et non l'inverse ${ }^{78}$. La «retraite», composée de l'usufruit des biens ou exprimée en termes «d'aliments et de nécessités », n'est pas une pratique exclusivement montpelliéraine, car elle est liée au système dotal et successoral ${ }^{79}$.

Et quand la planification des vieux jours d'une femme n'est pas prévue par son mari, elle peut être prise en charge par un autre membre de sa parenté. Peyre Cabassut, menuisier et père de famille, lègue en 1421 à sa mère Dulcia une couche (colga) garnie d'un édredon, de draps et d'un coussin, ainsi qu'un lit, qui se trouvent chez lui; l'épouse du testateur s'occupera de l'entretien de sa belle-mère ${ }^{80}$. Grâce aux aménagements demandés par le testateur, Dulcia est certaine de ne pas connaître les désagréments du grand âge dans l'isolement, puisqu'elle trouvera auprès de sa belle-fille les soins dont elle aura besoin jusqu'à ses derniers jours. La même année, Catherina, femme de Johan Serilhan, poissonnier de Montpellier, demande à ses enfants et son époux, nommés héritiers universels, d'entretenir sa mère Peyronela si celle-ci se trouve «in decrepitate» et ne peut plus «se jurare ${ }^{81} »$.

Le risque de la vieillesse-senium, synonyme de dépendance juridique, économique et physique, est prévenu par la mise en branle des solidarités et devoirs familiaux. Dans les compoix de Montpellier, la majorité des contribuables âgés vivent avec leurs enfants, filles ou garçons adultes et mariés, témoignage de l'investissement des relations de filiation dans la prise en charge de la vieillesse. À défaut, les Montpelliérains se tournent vers leurs collatéraux, faisant appel à leurs neveux et nièces pour prendre soin d'eux. Bérauda, veuve de Paul Privat, est mère de deux enfants, une fille mariée et un fils, nommé Gibert, avec lequel les relations sont très tendues. En public, il l'a rabrouée vertement et a porté la main sur elle ${ }^{82}$.

transmettre leurs biens à leurs enfants. Statistiques réalisées sur la base de 564 testaments datés des années 1250 à la fin du $\mathrm{Xv}^{\mathrm{e}}$ siècle.

77. $C f$. K. French, «Women in the Late Medieval English Parish», dans M. ErLer et M. KowALESKI éd., Gendering the Master Narrative. Women and Power in the Middle Ages, Ithaca, 2003, p. 156-173.

78. Les époux de ces femmes demandent souvent que la donation soit conditionnée au maintien du veuvage et l'assortissent de clauses de cohabitations avec les héritiers universels, l'un ou plusieurs des enfants du couple: $c f$. M.-T. LoRCIN, «Retraite des veuves et filles au couvent...».

79. Dans le Toulousain: $c f$. M.-C. MARANDET, «Le patrimoine des femmes... », p. 50.

80. Montpellier, ADH, 2 E 95-463, Arnaud Vitalis, 9 juillet 1421, fo 76.

81. Montpellier, ADH, 2 E 96-463, Arnaud Vitalis, 24 septembre 1421, fo 134: «Item volo et ordino quod causa quo domina mater mea veneret in decrepitate et non posset se jurare quod omnes tres teneant ipsam alimentare $\gg\left(f^{\circ} 135\right)$.

82. Montpellier, ADH, 2 E 95-445, Arnaud Vitalis, 13 mai 1412, fo $27:$ « $[\ldots]$ pro eo quod ipse Gibertus filius meus me verberavit acriter et manum ingessit super me in presencia Johannis Bort et Petri Capucii et Johannis Aufredi, fusterii et plurium aliorum » ( $\left.f^{\circ} 28\right)$. 
Dans son testament daté de 1412, Bérauda ne laisse que 30 sous à son fils, alors qu'elle transmet à sa nièce tous ses biens situés à Bredon, dans le diocèse de Saint-Flour dont elle est originaire, et fait de sa fille mariée son héritière universelle. La petite-nièce de la testatrice, Johannetta, recevra une maison quand elle se mariera, maison dans laquelle elle vit avec sa grandtante $^{83}$. Bérauda est veuve, sa fille a quitté le foyer et elle porte le poids de l'ingratitude de son fils; la testatrice compte alors sur sa petite-nièce, le don de la maison aidant, pour l'accompagner dans la vieillesse.

Se retirer de la vie active est plus aisé à faire lorsque l'on vit déjà avec ses descendants, ou quand il est possible d'emménager avec eux. Une relative aisance financière est un avantage indéniable pour les familles et les individus devant accueillir des personnes âgées. Les compoix nous permettent d'évaluer le niveau de richesse des habitants assujettis à l'impôt, car ils incluent une liste de leurs biens et leur estimation, exprimée en livres fiscales ${ }^{84}$. Il en ressort que les ménages élargis incluant des personnes âgés semblent un peu moins souffrir de la pauvreté que les autres et appartenir plutôt aux «classes moyennes» de la ville montpelliéraine, incarnée par les petits commerçants, les artisans et certaines professions libérales ${ }^{85}$. Les moyens dont ils disposent facilitent la vie commune en permettant l'achat de lits supplémentaires ou de disposer d'hostals assez grands pour accueillir une famille pluri-générationnelle.

83. «Item lego eodem jure institutionnis Johannete filie Johannis Segretis condam, nepte mee, filie Catherine nepte mee et uxoris dicti condam Johannis Segreti, quae Johanneta mecum commoratur de presenti; videlicet hospicium meum in quo habito et in quo moram traho [...] situm en extra muros communis closure ville Montispessulani, in carreria vocata La Soquaria, confrontatum [...liste des confronts...] quod legatum facio eidem Johannete pro suo maritagio ad omnimodas voluntates suas et suores » (ibid., $\left.\mathrm{f}^{\circ} 27 \mathrm{v}^{\circ}\right)$.

84. Il est très délicat de convertir ces sommes en livres réelles. On peut cependant traiter l'ensemble des estimations de manière cohérente, par rapport à elles-mêmes. Voir la thèse d'A.-C. Marin-Rambier, Montpellier à la fin du Moyen Âge d'après les compoix (13801450), Thèse de l'École nationale des chartes, 1980. Pour une illustration de cette approche, cf. L. LAumonier, Vivre seul à Montpellier..., chapitre 2.

85. Sur les métiers à Montpellier au Moyen Âge, $c f$. A. Gouron, La Règlementation des métiers en Languedoc, Paris, 1958. Sur les «classes moyennes», voir le cas toulousain dans P. WolfF, Les «Estimes» toulousaines des XIV et XVe siècles, Toulouse, 1956, p. 108 sq. 


\begin{tabular}{|l|l|l|}
\hline $\begin{array}{l}\text { Estimation des biens } \\
\text { (livres fiscales) }\end{array}$ & $\begin{array}{l}\text { Ménages élargis avec } \\
\text { personne âgée }(122)\end{array}$ & $\begin{array}{l}\text { Moyenne des déclarations } \\
(8858) *\end{array}$ \\
\hline Basse $(<50$ livres) & $43 \%(52)$ & $54,1 \%(4790)$ \\
\hline Moyenne (<250 livres) & $42 \%(51)$ & $28,3 \%(2505)$ \\
\hline Haute $(>250$ livres $)$ & $14 \%(17)$ & $11,5 \%(1019)$ \\
\hline Non déterminé & $2 \%(2)$ & $6,1 \%(544)$ \\
\hline
\end{tabular}

*Ce chiffre est inférieur au nombre total de déclarations fiscales (9347) car plusieurs déclarations n'incluent pas d'estimation des biens; certains registres sont inachevés et ne présentent aucune estimation; d'autres, très incomplets et datant des années 1446-1447 n'ont pas été pris en compte car ils ont donné lieu à une réfection en 1448-1449.

\section{Répartition des estimations des biens dans les compoix (1380-1480)}

Les ménages souche sont en moyenne davantage représentés dans les milieux aisés et riches de la société montpelliéraine. Peut-être peut-on y voir une trace de la tendance des familles notables à vivre dans des ménages très élargis ${ }^{86}$. Les vastes demeures des riches habitants de Montpellier ne manquent pas d'espace pour accueillir les ascendants, les collatéraux et les familiers, ainsi que le personnel domestique ${ }^{87}$. Pourtant, la pauvreté ne semble pas un obstacle majeur à ces cohabitations, puisqu'environ $40 \%$ des ménages élargis incluant une personne âgée ou vieillissante ne possèdent que des biens de maigre valeur et appartiennent aux milieux défavorisés de la ville ${ }^{88}$. Malgré une bouche supplémentaire à nourrir, les personnes âgées participent aux tâches domestiques quand elles ne peuvent plus travailler. Si faire vie commune ne permet pas d'échapper à la pauvreté, les personnes âgées peuvent se reposer sur les autres membres du ménage pour prendre soin d'elles. Elles ne mourront pas dans la solitude. Si les sources fiscales et les testaments montrent surtout l'image de personnes âgées entourées par leur famille, de nombreux Montpelliérains âgés sont privés de ces liens fondamentaux et ne pourront résider avec leurs proches pour leur fin de vie.

\section{La vieillesse sans parents}

La mortalité infantile, la stérilité, l'immigration et les épidémies de la fin du Moyen Âge expliquent la proportion élevée d'individus privés de

86. Cf. R. CARron, Enfant et parenté dans la France médiévale, $X^{e}$-XIII ${ }^{e}$ siècles, Genève, 1989, p. 27 ; A. BuRGiÈre et al. éd., Histoire de la famille, t. I, Paris, 1986, p. 396.

87. Une étude approfondie de l'habitat médiéval a été réalisée par B. SourNIA et J.L. Vayssettes, Montpellier, la demeure médiévale, Paris, 1991.

88. Les ménages estimés à moins de 25 livres sont considérés «nichils » par le consulat et exemptés d'une partie de l'impôt. Pour une étude de la répartition des allivrements au sein des contribuables, de 1380 à 1435, voir A.-C. MARIN-RAMBIER, Montpellier à la fin du Moyen Âge d'après les compoix... 
la présence d'enfants, confrontés au problème de préparer leur vieillesse et de ne pas mourir seuls. Dans les testaments, $45 \%$ des veufs et veuves et un quart des couples remariés sont sans descendants ${ }^{89}$. Certains contournent cet obstacle en faisant appel à d'autres membres de leur parenté pour prendre soin d'eux, comme on l'a vu. Mais, en l'absence de parents pouvant accueillir les personnes âgées, le patrimoine semble devenir déterminant dans la manière d'organiser la fin de vie, permettant de retenir auprès de soi des tiers.

\section{Des biens contre la solitude}

Le patrimoine des personnes âgées sans descendants leur permet d'attirer autour d'elles des individus chargés de les assister. Il s'agit en premier lieu du personnel domestique, employé pour prendre soin du vieillard et de sa demeure. L'étude des testaments montre que les personnes âgées ont recours à des serviteurs un peu plus souvent que les autres: environ $11,3 \%$ des testateurs jeunes et adultes emploient du personnel de maison, contre $18 \%$ des personnes âgées ${ }^{90}$. Marqueta, par exemple, veuve de Johan de Cugno et grand-mère, lègue à Johanna, sa servante, tous ses vêtements et bijoux ${ }^{91}$. Retenir auprès de soi des domestiques permet aux personnes âgées vivant seules de manifester leur niveau social, puisqu'elles ont les moyens de verser un salaire à une tierce personne et de conforter une position valorisante, ayant une certaine autorité vis-à-vis de leurs serviteurs, une potestas. L'embauche de personnel domestique est réservée aux Montpelliérains les plus aisés et ne constitue une assistance dans la vieillesse que pour une minorité d'individus. Cette pratique offre une certaine réciprocité, en permettant à des personnes vieillissantes, pauvres et isolées, de se placer au service d'un ménage, obtenant en échange le gîte et le couvert.

Ceux qui possèdent quelques terres ou une maison d'un peu de valeur peuvent faire cession de leurs biens en échange d'une vie commune et de leur entretien jusqu'à leur décès. Il s'agit d'un mécanisme semblable à celui étudié dans le cas de Peyre Perrucet, mais qui fait intervenir des personnes non apparentées. Estimé en 1404, Johan de Meyras possède une maison et des terres estimées à 60 livres fiscales ${ }^{92}$. Homme seul et vieillissant, il se porte encore bien et parvient à prendre soin de lui-même. Mais huit ans

89. Parmi 564 testaments, datés des années 1250 à la fin du Xve siècle. Sur 140 testaments de veufs et veuves, 63 n'ont pas d'enfant (45\%). Sur 43 testaments de personnes remariées, 11 n'ont pas d'enfant (26\%). Sur 295 testateurs mariés (première union), 119 (40\%) n'ont pas d'enfant.

90. 8 sur 44 .

91. Montpellier, ADH, 2 E 95-628, Jean Chavaleri (1460-1479), 28 juillet 1456, fo 27.

92. Montpellier, AM, Joffre 242, compoix de Saint-Mathieu, 1404, $\mathrm{f}^{\circ} 45 \mathrm{v}^{\circ}$. 
plus tard, la situation a changé et la senectus de Johan a fait place à une vieillesse débilitante: «venu en décrépitude» («vengutz ou decrepitat»), il s'est donné lui et ses biens à un certain Johan Telier, «que lo noyria ${ }^{93}{ }$. Si Johan de Meyras semble avoir attendu le dernier moment pour s'installer avec son donataire, d'autres Montpelliérains sans famille prévoient leur retraite avant d'avoir atteint un âge incapacitant. En 1421, Bernat Amans et Catherina, mari et femme, font un testament commun. Sans enfant et sans parenté consanguine, leurs héritiers universels sont Peyre Roman et sa femme Lucia, à charge pour eux d'assurer la subsistance et l'entretien de Bernat et Catherina leur vie durant, même s'ils sont «impotentes, senes et in valetudina ${ }^{94} \gg$.

Bernat et Catherina n'ont pas d'enfant en vie: leur testament ne montre l'existence d'aucun réseau si ce n'est ce couple, Peyre et Lucia. Par cette donation les testateurs préparent leur fin de vie, sachant que si l'un des deux vient à tomber malade et à décéder, l'autre n'affrontera pas seul la mort. Vieillir et mourir dans la solitude sont combattus par le recours à l'adoption ou à la donation ${ }^{95}$. Afin de s'assurer une fin de vie dans la dignité, au sein d'un foyer et d'écarter le spectre d'une senium en solitaire, les personnes vieillissantes et sans famille doivent chercher en dehors de leur parenté le soutien nécessaire. Les exemples peuvent être multipliés et se trouvent dans des actes de diverses natures : certains contrats, assimilables à des démarches d'adoption, donnent la possibilité aux Montpelliérains sans

93. «Lan MCCCXII a VIII de febrier los senhors [...liste des consuls...] attendut que lo dich Johan de Meyras es vengutz ou decrepitat et ses donatz a en Johan Telier, fayssier, que lo noyria et jura que non a degun cabal, lileveron de son manifest las quaranta 11 que lo dich Johan de Meyras era en manifest per cabal. Et agut sagramen del dich Johan Telier, de sa volontat lo abateron per les xviii 11 que monta los heretages del dich Johan de Meyras et per son moble per tot a vint et sinc 11. »

94. Montpellier, ADH, 2 E 95-463, Arnaud Vitalis (1421), 24 mai 1421, $\mathrm{f}^{\circ} 48-48 \mathrm{v}$ : «In omnibus vero et singulis aliis bonis meis tam mobilibus quam inmobilibus presentibus et futuris quocumque sint qualiacumque quantacumque et quomodocumque, nuncupentur heredes meos universales facimus et institamus [sic] et ore proprio nominamus Petrum Roman sabaterium et Luciam ejus uxorem equis partibus [...]. Et hanc institutionem hereditariam facimus ad fratres ut teneantur nobis et cuilibet $[\ldots]$ alere, nutrire et alimentare tam quantum vixerimus in hoc mundo cum nos simus impotentes et senes et in valetudina [sic]. » Peyre Roman et sa femme Lucia reconnaissent la donation dans un acte séparé et acceptent les conditions. Ibid., 24 mai 1421, fo 48v-49: «Nos Petrus Roman sabaterius et Lucia conjuges Montispessulani [...] promictimus [sic] vobis dictes Bernardo Amans et Catherine, conjugibus, ibidem presentibus etc. alere, nutrire et alimentare bene et honeste juxta nostrum statuum tam quantum vitam duxerimus in hoc mundo. »

95. «N'est-ce pas simplement la crainte de la solitude, de mourir seul et sans soutien que livrent ces clauses d'adoption, d'affiliation et de cohabitation?»: A. COuRTEMANCHE, «Lutter contre la solitude: adoption et affiliation à Manosque au Xv siècle», Médiévales, 19 (1990), p. 37-42 (p. 41). 
enfant de s'assurer une vieillesse et une mort en compagnie ${ }^{96}$. Mais ces donations ne sont possibles que quand on dispose de quelques biens. La grande pauvreté ne permet pas d'inciter quelqu'un à faire vie commune, surtout quand la dépendance guette les individus, devenus des charges pour autrui. Prendre sa retraite est un luxe que ne peuvent s'accorder les personnes âgées pauvres et isolées ${ }^{97}$.

\section{Vieillir en solitaire : pauvreté et vulnérabilité}

Il est fondamental pour ceux qui ne disposent pas de rentes de maintenir une activité aussi longtemps que possible, à la fois pour des raisons financières évidentes et pour conserver un statut social valorisé de personne active, «en capacité ${ }^{98}$ », occupant un rôle social ${ }^{99}$. De plus en plus rares avec le grand âge, les gains du travail sont bien maigres dans le cas des femmes. Si de nombreuses habitantes de la ville travaillent tout au long de leur vie, leurs tâches se déroulent souvent «dans l'ombre», de manière informelle; elles sont moins qualifiées que les attributions masculines et bien moins rémunérées ${ }^{100}$. Pauvreté, vieillissement et isolement vont ainsi de pair avec le maintien forcé d'un métier, comme en témoignent les compoix. La totalité des hommes décrits comme «vieux» ou «impotents » et qui sont sans soutien familial déclarent une activité professionnelle, tandis que moins d'un tiers des hommes âgés qui habitent en compagnie le font. Les registres fiscaux montrent avec acuité les difficultés économiques rencontrées par les vieillards isolés de la ville.

96. Sur l'adoption au Moyen Âge, voir le numéro 35 de la revue Médiévales, consacré à ce sujet, en particulier D. LETT, «Droits et pratiques de l'adoption au Moyen Âge», Médiévales, 35 (1998), p. 5-8. Des études très récentes sur les formes de l'adoption en Italie à la fin du Moyen Âge et à l'époque moderne, voir la section «Pratiche dell'adozione in età bassomedievale e moderna », Mélanges de l'École française de Rome. Italie et Méditerranée, 124/1 (2012), p. 121-271; pour un bilan, $c f$. M. GarbellotTI et M. C. Rossi, « Et deliberaverunt acceptare eum per suum filium adoptivum. Pratiche dell'adozione e dell'affidamento in età medievale e moderna », ibid., p. 121-125. Pour des exemples montpelliérains : $c f$. Montpellier, ADH, 2 E 95-472, Arnaud Vitalis (1433), 6 janvier 1433 (a.s.), fo 353v; 2 E 95-538, Giraud Girard (1434-1436), 13 avril 1434, $\mathrm{f}^{\circ} 19$. Pour une analyse de ces actes, $c f$. L. LAUMONIER, «Manières de parenté: les formes de l'adoption dans la région de Montpellier au XV siècle», Annales du Midi, 289 (2015), p. 5-22.

97. P. Thane, Old Age in English History..., p. 90-92 ; C. GAUVARD, « De grace especial» Crime, État et société en France à la fin du Moyen Âge, Paris, 1991 (2010), p. 370-371.

98. I. Metzler, A Social History of Disability..., p. 111 sq.

99. Pour une comparaison avec la situation en Italie, $c f$. D. Herlihy, «Age, Property and Career in Medieval Society », dans M. M. Sheehan éd., Aging and the Aged..., p. 143-158.

100. $C f$. C. BÉGHIN-LE GourRIÉREC, «Entre ombre et lumière, quelques aspects du travail des femmes à Montpellier (1293-1408)», Médiévales, 30 (1996), p. 45-54; ID., «Donneuses d'ouvrages, apprenties et salariées aux XIV et $X^{\mathrm{e}}$ siècles dans les sociétés urbaines languedociennes », Clio, 3 (1996), p. 31-54. 
En 1435, quatre résidents du quartier de Sainte-Croix sont décrits comme âgés. Tous vivent seuls et sont dans une grande pauvreté. Le premier est Guilhemin Guilhem, lavandier, exempté pour pauvreté, car il est «vielh e impotent ${ }^{101} \gg$. Le deuxième est une femme, dona Catarina Garestanha, qui a une maison et une carterée de vigne, mais qui «es impotent e mot paura e vielha ${ }^{102} »$. Ses biens ne valent que 13 livres fiscales, ce qui l'inclut dans la catégorie des «nichils », ceux trop pauvres pour payer l'impôt. Ensuite vient dona Guilhalma Peyroza, qui est d'emblée dite «paura». Le notaire ajoute qu' «es vielha, despoderada e mot paura, e juret a XV de mars 1434 que non a moble ne autra causa ${ }^{103} »$. Pour finir, Peyre de Polssan, forgeron, «vielh e paure », prête serment de ne rien posséder ${ }^{104}$. L'étude de la répartition des richesses fait écho à ces cas individuels : les deux tiers des personnes âgées seules des compoix vivent dans la pauvreté.

\begin{tabular}{|l|l|l|}
\hline $\begin{array}{l}\text { Estimation des biens } \\
\text { (livres fiscales) }\end{array}$ & Personne âgée seule (33) & $\begin{array}{l}\text { Ensemble des déclarations } \\
(8858)\end{array}$ \\
\hline Basse $(<50$ livres) & $67 \%(22)$ & $54,1 \%(4790)$ \\
\hline Moyenne (<250 livres) & $30 \%(10)$ & $28,3 \%(2505)$ \\
\hline Haute $(>250$ livres $)$ & - & $11,5 \%(1019)$ \\
\hline Non déterminé & $3 \%(1)$ & $6,1 \%(544)$ \\
\hline
\end{tabular}

\section{Répartition des richesses entre les personnes âgées seules (1380-1480)}

Et, alors que $14 \%$ des ménages élargis comptant une personne âgée appartiennent aux milieux aisés, aucune personne âgée vivant seule ne possède de biens valant plus de 250 livres fiscales. La surreprésentation des personnes âgées isolées dans les rangs des pauvres montpelliérains est un indicateur de leur vulnérabilité. En tant que pauvres, elles appartiennent aux nombreuses miserabiles personae de Montpellier, vivant de la charité et des aumônes ${ }^{105}$. Le sort malheureux des contribuables âgés est reconnu par le consulat de la ville qui manifeste sa charité à leur égard. En 1411, les consuls réduisent l'estimation de Jacme Baquier, «car il est décrépi de

101. Montpellier, AM, Joffre 251, compoix de Sainte-Croix, 1435, f ${ }^{\circ} 86$.

102. Ibid., f $\mathrm{f}^{\circ} 104 \mathrm{v}^{\circ}$.

103. Ibid., f ${ }^{\circ} 105$.

104. Ibid., $\mathrm{f}^{\circ} 110 \mathrm{v}^{\circ}$.

105. «[...] the term pauper in early medieval texts, up really to the eleventh century, implies lack of power and effectiveness more truly than lack of material possessions. [...] We must wait, it seems, until the late Middle Ages to find clear and forceful assertions of a linkage between advancing years and deepening poverty» (D. HeRLIHY, «Age, Property and Career...»,p. 144). 
sa personne, et ne peut rien gagner [en travaillant] ${ }^{106} »$. Bien qu'appauvri par son incapacité à gagner sa vie, Jacme possède encore quelques biens qui lui permettront de subsister. Ce double processus de vieillissement et d'appauvrissement est perceptible dans la déclaration fiscale de Peyre Dandura, abattu de 130 livres en 1409, car «es vengutz en decrepit de sa persona et en pauretat ${ }^{107} »$. L'emploi du verbe «venir» montre l'existence d'un mouvement, d'une régression conjointe de l'état de santé et de pauvreté de la personne. La vieillesse grandissant, les ressources s'amenuisent, rendant d'autant plus vulnérables les individus isolés.

La mendicité et la marginalisation guettent ces personnes âgées, isolées dans le centre urbain. C'est peut-être pour cette raison qu'en 1390 Peyre de la Foras, pâtissier veuf, lègue à Dulcia, veuve d'un autre pâtissier, Raymond de Cruoyas, son entretien complet: nourriture et boisson, vêtements et souliers, logement, sa vie durant ${ }^{108}$. Si Dulcia décède dans la maison de Raymond, alors il lui offre en sus 20 florins d'or, pour qu'elle puisse tester. Si, en revanche, la veuve ne parvient pas à cohabiter en paix avec le fils de Peyre, Bertholomieu et avec l'épouse de ce dernier, alors elle recevra 100 florins, une somme très généreuse, qui lui permettra d'assurer sa subsistance ${ }^{109}$. Les liens entre Dulcia et Peyre sont sans doute nés des sociabilités professionnelles, puisque son mari était pâtissier, métier que pratique le testateur. Dulcia et Peyre sont tous deux veufs mais, parce que Dulcia n'a pas de famille, sa vieillesse s'annonce difficile, ses ressources semblant maigres au vu des dons de subsistance qu'elle reçoit. Peyre veille à ce que Dulcia ait un toit, des vêtements et des aliments jusqu'à sa mort, même dans la maladie, sans aucune contrepartie. Bien que la vie urbaine puisse éroder les relations familiales, elle est aussi le lieu des solidarités nées de l'amitié, du voisinage ${ }^{110}$. Les villes suscitent aussi l'émergence et la multiplication de formes particulières de structures charitables, visant à remédier au problème social posé par la vieillesse vulnérable.

106. «car es decrepitat de sa persona, que non pot res gazanhar» (Montpellier, AM, Joffre 239, compoix de Saint-Firmin, 1404, f $^{\circ} 142$ ).

107. Ibid., f ${ }^{\circ} 157$.

108. Montpellier, ADH, 2 E 95-390, Pierre Bourdon, 20 septembre 1390, $f^{\circ} 47$ : «Item lego Dulsie relicte Raymundi de Cruoyas condam pasticierii montispessulani victum suum cibi et potus et vestitum, secundum statum persone sue, in et super lucro faciendo in hoperatorio meo » $\left(f^{\circ} 48 v^{\circ}\right)$.

109. «Si dicta Dulcia non se posset convenire seu concorderre [sic] cum Bartholomeo de Somilhaco herede meo infrascripto et uxore sua, et se abeis dissedere vellet in dicto casu dicte Dulcie lego centum florenes auri boni ponderis» (ibid.).

110. Montpellier, ADH, 2 E 95-441, Arnaud Vitalis (1409), 2 septembre 1409, fo 56 : Testament de Braydeta, veuve pauvre et sans famille, qui n'effectue que deux legs, à ses deux voisines, et institue un clerc son héritier universel. 
Les nombreux hôpitaux émaillant l'espace urbain, une dizaine aux $\mathrm{XIV}^{\mathrm{e}}$ et $\mathrm{XV}^{\mathrm{e}}$ siècles, sont les principales structures charitables de la ville $^{111}$, en ouvrant leurs portes à l'ensemble des pauvres malheureux, voyageurs, malades, et personnes âgées sans soutien ${ }^{112}$. La misère est bien le dénominateur commun à tous les «patients» des hôpitaux médiévaux. Quand la pauvreté empêche les vieillards de convaincre quelqu'un de prendre soin d'eux, les hôpitaux de Montpellier sont des recours appréciables pour effectuer de brefs séjours ou pour venir y mourir. Entourés par la communauté hospitalière, les personnes âgées sont certaines de recevoir les derniers sacrements et d'être ensevelies avec dignité. Les hôpitaux accueillent aussi des pensionnaires, qui ont donné leurs biens à l'institution et y résident, y finissant leur vie ${ }^{113}$. Ce système de pensionnat est une vocation de l'hôpital médiéval, car il s'agit là d'une forme d'assistance, religieuse, par l'expression des œuvres de miséricorde, et sociale, en prenant en charge une partie des personnes âgées ${ }^{114}$. Cependant, fort peu de sources permettent de connaître l'identité de ceux qui occupent les lits des hôpitaux de Montpellier et d'y affirmer la présence de personnes âgées ${ }^{115}$.

De manière plus ponctuelle, les confréries de dévotion, nombreuses à Montpellier, apportent une forme de soutien aux personnes âgées, en particulier dans leur rôle d'accompagnement de la mort ${ }^{116}$. La sociabilité qui règne entre les confrères ne doit pas être exagérée, ce dont témoignent les testaments: si 17,5\% de l'ensemble des testateurs font un legs à une ou plusieurs confréries, aucun ne nomme de consœur ou de confrère ${ }^{117}$.

111. Les quatre principaux sont les plus anciens: Saint-Esprit, Notre-Dame/Saint-Éloi, Saint-Jacques et la léproserie Saint-Lazare de Castelnau; $c f$. A. Germain, «De la charité publique et hospitalière à Montpellier au Moyen Âge», Mémoires de la société archéologique de Montpellier, 4/27 (1859), p. 482-552; L. DuLIEu, Histoire de la médecine à Montpellier : le Moyen Âge, Avignon, 1975; G. Dumas, Santé et société à Montpellier à la fin du Moyen Âge (1293-1516), Leyde, 2014.

112. Cf. Daniel Le Blévec (La Part du pauvre..., «Les assistés», p. 771-788) en propose une typologie: les hôpitaux accueillent les pauvres passants, sans domicile fixe ou voyageurs, les pèlerins, les pauvres malades, les femmes enceintes, les vieillards.

113. Cf. P. Thane, Old Age in English History..., p. 81-83. Pour une étude de cas, cf. A. SAUNIER, «Une fin de vie: Jeanne la Grigète à l'hôpital du Saint-Esprit de Paris, 1434», dans H. Dubois et M. Zink éd., Les Âges de la vie..., p. 269-287.

114. Cf. D. Le Blévec, La Part du pauvre..., p. 783.

115. Huit testateurs y dictent leurs dernières volontés mais aucun ne semble particulièrement âgé. Entre autres: Montpellier, ADH, 2 E 95-450, Arnaud Vitalis, 27 août $1434, \mathrm{f}^{\circ} 69 \mathrm{v}^{\circ} ; 2$ E 95-538, Giraud Girard, 31 avril 1434, $\mathrm{f}^{\circ} 33$ et 7 avril $1434, \mathrm{f}^{\circ} 10 \mathrm{v}^{\circ} ; 2$ E 95-538, Jean Valocière l'Aîné, 31 août 1478, fo 102.

116. Pour une liste de ces confréries, $c f$. L. Laumonier, Vivre seul à Montpellier..., annexe $\mathrm{V}$.

117. Pour A. Vauchez (Les Laïcs au Moyen Âge. Pratiques et expériences religieuses, Paris, 1970, p. 118-119), les confréries ne constituent pas tant des structures de sociabilité 
L'importance des confréries dans les structures charitables de Montpellier s'affirme par le soutien offert pendant la maladie et surtout par l'encadrement de la mort, «terrain d'élection de l'action caritative confraternelle ${ }^{118}{ }$. Les statuts de la confrairie de monsenhour Sainct Jaume par exemple, datés de la fin du XIII ${ }^{\mathrm{e}}$ siècle, normalisent les gestes de charité et de solidarité entre les membres ${ }^{119}$. Quand un confrère ou une consœur est malade, les prévôts de la confrérie doivent lui rendre visite ${ }^{120}$. Les confrères accompagnent les membres défunts jusqu'à leur sépulture, assistent à la messe, prient pour leur âme ${ }^{121}$. Le chapelain de la confrérie chante pour les décédés pendant neuf jours après leur décès ${ }^{122}$.

En l'absence de famille pour accompagner le cortège funèbre et de revenus pour obtenir quelques messes, l'appartenance à une confrérie est la garantie de funérailles honorables et de prières pour le salut de l'âme du défunt. Parmi les testateurs âgés, $18 \%$ font un legs à la confrérie ou aux confréries dont ils sont membres, une proportion légèrement supérieure à la moyenne. Certains adhèrent à de nombreuses associations confraternelles, comme Maria Ganavoria, veuve vieillissante, consœur des confréries Saint-Jean de l'église Saint-Firmin, Saint-Germain et Saint-Blaise de l'église Saint-Germain, Sainte-Marie, Sainte-Croix et Sainte-Catherine de l'église Sainte-Croix ${ }^{123}$. Pas moins de six confréries participeront au cortège funéraire de Maria et prieront pour son âme pendant et après ses funérailles. Cette testatrice n'est pas une femme isolée; elle a plusieurs neveux et nièces, petits-neveux et petites-nièces qui pourront veiller sur elle pendant ses derniers instants. D'autres testateurs âgés en revanche sont bien moins entourés; leurs confrères constitueront sans nul doute les principaux présents lors des funérailles et les premiers intercesseurs priant pour le salut de l'âme du défunt.

Dans la crainte de la vieillesse-senium, les Montpelliérains effectuent des legs et des donations visant à protéger leurs aînés ou à préparer leur propre fin de vie. Ces actes manifestent une inquiétude face à la vieillesse

qu'un «phénomène de convivialité relativement banal».

118. C. VINCENT, Les Confréries médiévales dans le royaume de France, XIII'-XVe siècle, Paris, 1994, p. 77.

119. Ils sont édités dans A. GERMAIN, Histoire de la commune de Montpellier, Montpellier, 1854, t. III, pièce justificative XXXVI, p. 482 sq.

120. Article 4 et Article 9, ibid., p. 482 et p. 484.

121. Article 4, ibid., p. 482-483.

122. Article 11, ibid., p. 484.

123. Montpellier, ADH, 2 E 95-379, Pons Esmeric (1369-1402), 7 janvier 1369 (a.s.), $f^{\circ} 3 v^{\circ}$. 
vécue solitaire et montrent que les Montpelliérains veillent à ce que les aînés et eux-mêmes aient une place au coin du feu, dans un ménage. Cette place est parfois celle du détenteur ou de la détentrice de la patria potestas, à la tête du foyer domestique, parfois celle d'une personne en retrait, prise en charge, qui s'est écartée, ou a été écartée, en raison de son état ou pour laisser plus d'espace aux jeunes. Quand les individus n'ont pas de parenté vers laquelle se tourner pour leur vieillesse, les relations amicales et professionnelles, la fortune et les institutions d'assistance offrent un recours bienvenu contre la solitude. L'image parfois négative des personnes âgées isolées dans les textes prescriptifs et dans la littérature constitue peut-être une incitation à la vie en communauté familiale. C'est un rappel au devoir de solidarité entre les générations et un moyen de lutter contre l'isolement social des personnes âgées. Comme l'affirme Claude Gauvard, à la fin du Moyen Âge, «l'équilibre entre les générations est un des meilleurs moyens de sauvegarder l'ordre social ${ }^{124} \gg$. Dans une société où chacun a une place bien définie, la vieillesse solitaire pose le problème de la fragilité croissante des personnes âgées, de leur précarité économique et de la perte de leur rôle social. Ainsi, les solidarités familiales demeurent puissantes à Montpellier, que l'on soit riche ou pauvre. C'est en leur absence que la question des revenus des personnes vieillissantes et âgées se pose comme un problème, engendrant leur vulnérabilité. D'autres formes de solidarités, amicales, hospitalières ou encore confraternelles, peuvent parfois pallier ces problèmes. Mais les usages que les personnes font de cet ensemble de recours sont propres aux situations individuelles - les actes en témoignent et aux contraintes auxquelles chacun est confronté ${ }^{125}$.

124. C. GAUVARD, «De grace especial »..., p. 382.

125. Merci au professeur Didier Lett (Université Paris 7) et à la professeure Sharon Farmer (University of California, Santa Barbara, États-Unis), ainsi qu'aux évaluateurs de Médiévales, pour leurs conseils et leurs commentaires sur cette recherche, menée grâce à une bourse postdoctorale du Fonds de recherche du Québec - Société et culture (FRQSC), à l’Université du Minnesota (États-Unis). 
Lucie Laumonier - University of Minnesota, Minneapolis

\section{En prévision des vieux jours : les personnes âgées à Montpellier à la fin du Moyen Âge}

L'histoire de la vieillesse et des personnes âgées à la fin du Moyen Âge s'appuie surtout sur l'analyse des textes et constitue encore principalement une histoire de leurs représentations, plus qu'une étude de leur place dans la société. À travers les nombreuses sources de la ville de Montpellier, cette recherche vise à définir la vieillesse au regard des archives de la pratique, d'y saisir et d'y évaluer la présence des personnes âgées, de comprendre quelles alternatives leur sont offertes pour prévoir leurs vieux jours et d'identifier les obstacles qu'elles rencontrent éventuellement au cours de ce processus. Deux variables principales semblent peser sur l'organisation de la fin de vie : la présence ou l'absence d'une parenté sur laquelle se reposer et le niveau de richesse de l'individu. De la personne âgée aisée et entourée à l'individu pauvre et isolé se déclinent de multiples situations, témoignage des différentes de manières de vieillir dans un centre urbain méridional à la fin du Moyen Âge.

Famille - histoire urbaine - Montpellier - pauvreté - vieillesse

\section{Preparing for Old Age : The Elderly in Montpellier at the End of the Middle} Ages

Late medieval history of old age and of the elderly relays mainly on texts analysis and remains a history of their representations, more than a study of their place within the society. Based on many sources from the city of Montpellier, this paper aim to define old age in the archival document, to evaluate the presence of the elderly, to understand in which ways they can plan their old days, and to identify the limitations they face in this process. Two elements seem to influence the organization of old days : having or not family members to relay on, and the socioeconomic background of each individual. From the rich elderly surrounded by family, to the poor and lonely ones, many situations can be declined that all evocate the different ways of ageing in city at the end of the Middle Ages.

Family - Montpellier - Old Age - Poverty - Urban History 



\section{Langues d'Angleterre,}

coordonné par Alban Gautier et Jean-Pascal Pouzet

5 Alban Gautier et Jean-Pascal Pouzet

Les langues de

l'Angleterre médiévale : au-delà du bilinguisme

\section{David Trotter}

Peut-on parler de judéoanglo-normand?

Textes anglo-normands en écriture hébraíque

35 Christopher Lucken

Le beau français

d'Angleterre. Altérité de l'anglo-normand et invention du bon usage

57 Aude Mairey John Gower ou le multilinguisme en action

73 Catherine Nall et Daniel Wakelin Le déclin du multilinguisme dans The Boke of Noblesse et son Codicille de William Worcester
Essais et recherches

93 Donatella Nebbiai

Les livres de Jean

Durand († 1416),

"physicien »

et astrologue

\section{Lucie Laumonier}

En prévision des vieux

jours: les personnes âgées à Montpellier à la fin du Moyen Âge

\section{Points de vue}

147 François Foronda Procès politiques: une manie française?

161 Clément Lenoble Monnaie, valeur et citoyenneté chez Olivi et Eiximenis. "Moralisation de l'économie » ou "économie politique" médiévale?

181 Notes de lecture 205 Livres reçus 